\title{
Mutual Energy Transfer in a Binary Colloidal Quantum Well Complex
}

\author{
Junhong Yu, ${ }^{\dagger}$ Manoj Sharma, ${ }^{\dagger, \uparrow \odot ~ S a v a s ~ D e l i k a n l i, ~}{ }^{\dagger, \$}$ Muhammad Danang Birowosuto, ${ }^{\S}{ }^{\circ}$ \\ Hilmi Volkan Demir, $*,+,+, \| \odot$ and Cuong Dang $*, t, \S_{\odot}$
}

${ }^{\dagger}$ LUMINOUS! Centre of Excellence for Semiconductor Lighting and Displays, School of Electrical and Electronic Engineering, The Photonics Institute (TPI), Nanyang Technological University, 50 Nanyang Avenue, 639798 Singapore

${ }^{\ddagger}$ Department of Electrical and Electronics Engineering and Department of Physics, UNAM-Institute of Materials Science and Nanotechnology, Bilkent University, Bilkent, Ankara 06800, Turkey

${ }^{\S}$ CINTRA UMI CNRS/NTU/THALES 3288, Research Techno Plaza, 50 Nanyang Drive, Border X Block, Level 6, 637553 Singapore

"School of Physical and Mathematical Sciences, Division of Physics and Applied Physics, Nanyang Technological University, 639798 Singapore

\section{Supporting Information}

ABSTRACT: Förster resonance energy transfer (FRET) is a fundamental process that is key to optical biosensing, photosynthetic light harvesting, and downconverted light emission. However, in total, conventional FRET in a donoracceptor pair is essentially unidirectional, which impedes practical application of FRET-based technologies. Here, we propose a mutual FRET scheme that is uniquely bidirectional in a binary colloidal quantum well (CQW) complex enabled by utilizing the $d$ orbital electrons in a dopant-host CQW system. Steady-state emission intensity, time-resolved, and photoluminescence excitation spectroscopies have demonstrated that two distinct CQWs play the role of donor and acceptor simultaneously in this complex consisting of 3 monolayer (ML) copperdoped CQWs and 4 ML undoped CQWs. Band-edge excitons in 3 ML CQWs effectively transfer the excitation to excitons in $4 \mathrm{ML}$ CQWs, whose energy is also harvested backward by the dopants in $3 \mathrm{ML}$ CQWs. This binary CQW complex, which offers a unique mutual energy-transfer mechanism, may unlock revolutionary FRET-based technologies.

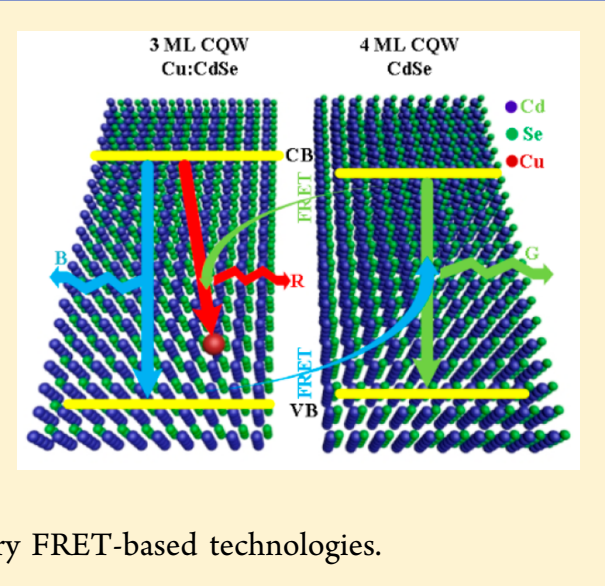

$\mathrm{F}$ örster resonance energy transfer (FRET), which describes the nonradiative energy transport via dipolar coupling from a donor to an acceptor, ${ }^{1,2}$ is a powerful tool used in various domains spanning from biophysics, ${ }^{3-5}$ for detecting inter/intra-molecular interactions at nanometer-scale, to energy optoelectronics, ${ }^{6-8}$ for enhancing light-conversion or light-harvesting via modulating FRET rate. In biophysics, the dynamics in biomolecules of interest can be quantified by monitoring the FRET rate between a labeled donor-acceptor pair. ${ }^{9,10}$ However, conventional FRET in a binary complex is a one-way process and offers only one output information. This makes the interpretation of potential dynamics not comprehensive or even inaccurate, because the rate of FRET depends on a number of factors, ${ }^{2,11,12}$ including the distance between the donor-acceptor pair, the spectral overlap between the donor emission and the acceptor absorption, the quantum efficiency of the donor, and the dielectric environment. On the other hand, control of FRET is always at the forefront of energy optoelectronics. In recent years, multiple external stimuli, such as temperature, ${ }^{13}$ electric field, ${ }^{12}$ the local density of states (LDOS), ${ }^{14,15}$ or Coulomb screening, ${ }^{11}$ have been successfully adopted to control FRET in nanomaterial complexes. Unfortunately, such conventional FRET with intrinsically unidirectional energy transfer restricts our control capability for multiparameter systems, e.g., light-conversion among red, green, and blue colors. ${ }^{16,17}$

Colloidal quantum wells (CQWs), ${ }^{18-21}$ owing to their high quantum efficiency, narrow emission spectrum, and solution processability, have recently evoked considerable attention in FRET studies. ${ }^{22-24}$ Several unique properties have indicated CQWs rather than other lower-dimensional nanomaterials (like OD and 1D) as ideal FRET donors and acceptors. Specifically, the extended 2D geometry which enhances the dipole interactions ${ }^{22,25}$ and atomic thickness control in the quantum-confined dimension which reduces the possibility of homo-FRET ${ }^{26}$ ensure the binary CQW complex achieves nearunity FRET efficiency ${ }^{13}$ and an Auger-outpaced FRET rate. ${ }^{22}$ It is worth noting that the significant development of transition metal ion-doped CQWs (e.g., $\mathrm{Ag}, \mathrm{Cu}$, or $\mathrm{Mn}$ ) over the past few years has led to a new possibility for FRET in a binary CQW complex. ${ }^{27-30}$ Metal ion dopants, which introduce midgap energy levels and radiatively capture the conduction band electrons, can generate a new emission band with a huge Stokes shift. ${ }^{31,32}$ An appealing conjecture in the binary

Received: July 5, 2019

Accepted: August 14, 2019

Published: August 21, 2019 

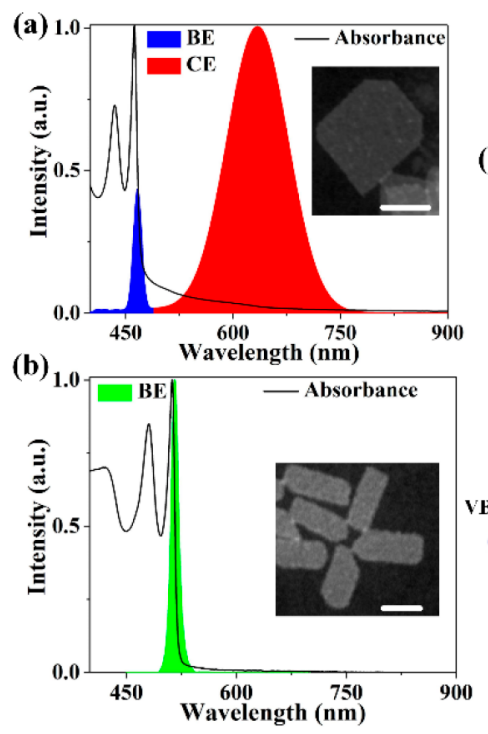

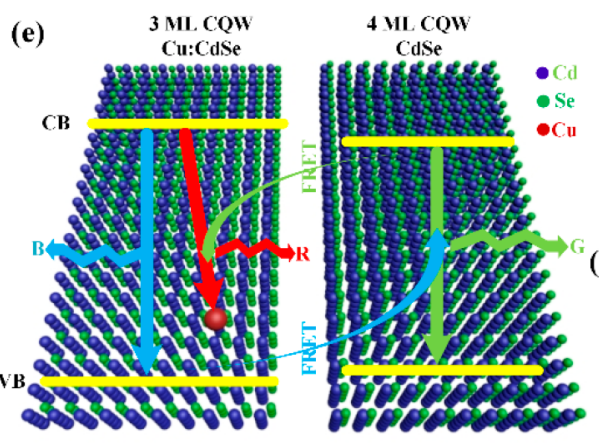

(c)

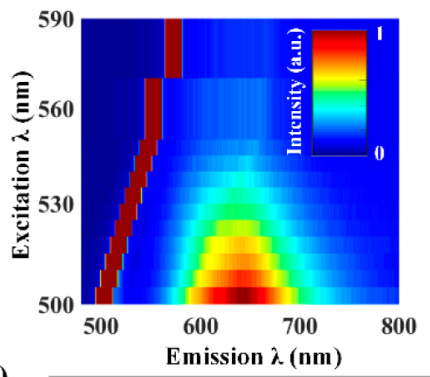

d)

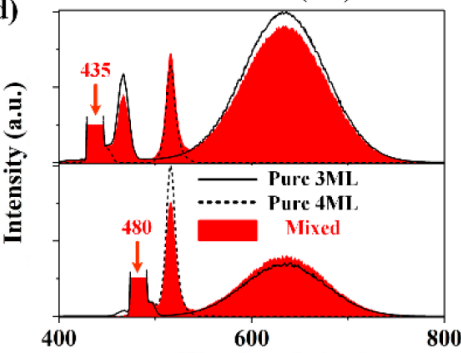

Figure 1. Static characterizations of $3 \mathrm{ML}$ d-CQWs and $4 \mathrm{ML}$ u-CQWs for FRET. (a) Absorption (black solid line) and emission spectra of $3 \mathrm{ML}$ copper-doped CQWs dissolved in hexane. The band edge emission (BE) is shaded in blue, and copper-related emission (CE) is shaded in red. Inset: a TEM image of $3 \mathrm{ML}$ d-CQWs; scale bar, $20 \mathrm{~nm}$; the lateral size of $3 \mathrm{ML} \mathrm{d-CQWs} \mathrm{is} \sim(45 \mathrm{~nm} \times 41 \mathrm{~nm})$. (b) Absorption (black solid line) and emission spectra of $4 \mathrm{ML}$ undoped CQWs dissolved in hexane. The band edge emission (BE) is shaded in green. Inset: a TEM image of $4 \mathrm{ML}$ u-CQWs; scale bar, $20 \mathrm{~nm}$; the lateral size of $4 \mathrm{ML}$ u-CQWs is $\sim(41 \mathrm{~nm} \times 16 \mathrm{~nm})$. (c) Normalized 2D emission map of $3 \mathrm{ML}$ d-CQWs dissolved in hexane under excitation with progressively lower photon energy. The excitation light (brown; maximum intensity is cut at 1 because of the much higher intensity) is also included for reference. (d) Comparison of the steady-state PL between CQW complex and pure component. The molar concentration ratio between $\mathrm{u}-\mathrm{CQWs}$ and d-CQWs is 1:10. All samples are in a solid film. Top panel: excitation light peaks at $435 \mathrm{~nm}$, corresponding to the light-hole electron transition in $3 \mathrm{ML}$ d-CQWs. Bottom panel: excitation light peaks at $480 \mathrm{~nm}$, corresponding to the lighthole electron transition in $4 \mathrm{ML}$ u-CQWs. (e) Schematic illustration of the potential mutual energy transfer in the CQW complex; CB, conduction band; VB, valence band. The values of energy edges of the $\mathrm{CB}$ are $-3.20 \mathrm{eV}(3 \mathrm{ML})$ and $-3.41 \mathrm{eV}(4 \mathrm{ML})$, and the values of energy edges of the $\mathrm{VB}$ are $-5.87 \mathrm{eV}(3 \mathrm{ML})$ and $-5.82 \mathrm{eV}(4 \mathrm{ML})$. The energy level of copper dopants distributes in a wide range; here, for clarity, we present only the highest-density states. R, G, and B denote the three primary colors, respectively. They correspond to three radiative transitions: CE of d-CQWs, $\mathrm{BE}$ of $\mathrm{u}-\mathrm{CQWs}$, and BE of d-CQWs.

complex consisting of doped and undoped CQWs is that if the metal ions can also behave as acceptors, FRET back and forth between doped and undoped CQWs can offer extended capability for FRET-based applications.

Herein, encouraged by the new FRET possibility induced by dopants and high FRET efficiency in CQWs, we have explored energy transfer between copper-doped 3 ML CQWs (dCQWs) and undoped $4 \mathrm{ML}$ CQWs (u-CQWs). For the first time in a binary inorganic colloidal nanomaterial complex, mutual FRET is unambiguously observed by the steady-state emission intensity, time-resolved PL (trPL), and photoluminescence excitation (PLE) spectroscopies. Because the $d$ electrons in $\mathrm{Cu}^{+}$can be directly excited into the conduction band of the CQW host, the d-CQWs and u-CQWs play the role of donor and acceptor simultaneously in the complex. The experimental results show that the exciton energy in $3 \mathrm{ML} d-$ CQWs is effectively transferred to the $4 \mathrm{ML}$ u-CQWs with an efficiency up to $\sim 50 \%$. Meanwhile, the exciton energy in $4 \mathrm{ML}$ $\mathrm{u}-\mathrm{CQWs}$ is harvested backward by the copper dopants in $3 \mathrm{ML}$ d-CQWs with a lower efficiency $(<10 \%)$ due to the small absorption cross section of dopant transition. ${ }^{29,30}$ Moreover, three emission bands from the CQW complex match the three primary colors (red, green, and blue) for human visual perception, which further enhances the favorability for practical applications. Our binary CQW complex, which presents mutual FRET beyond the unidirectional limitation in conventional systems and exhibits superior emission abilities, may provide a new design strategy for quantifying biophysical phenomena and constructing controllable lighting schemes.
In this work, copper-doped CdSe CQWs with $3 \mathrm{ML}$ vertical thickness and undoped CdSe CQWs with $4 \mathrm{ML}$ vertical thickness are selected as the donor-acceptor pair. Both the dCQWs and $\mathrm{u}$-CQWs are synthesized using modified recipes from our previous work, ${ }^{27,28,33}$ and the materials characterization is detailed in our previous work. ${ }^{27,28,32}$ The details of the synthesis and material characterization are described in Methods. Two basic requirements for FRET to occur are spatial and energetic overlap. ${ }^{2,22}$ The former implies the distance between a donor-acceptor pair should be smaller than the Förster radius, and the latter means spectral overlap between the donor emission and the acceptor absorption should be nonzero. The spatial overlap is easily satisfied in the CQW complex because of the extended plate geometry and large absorption cross section $\left(>10^{-14} \mathrm{~cm}^{2}\right)$ as detailed previously. ${ }^{18,22,33}$ In fact, the Förster radius for the CQW donor-acceptor pair $^{23}$ has been measured to be larger than 10 $\mathrm{nm}$. Here, we will mainly focus on the energetic overlap to explore the possible FRET in this two-component CQW complex.

The absorbance and PL spectra of d-CQWs (Figure 1a) show that the electron-heavy-hole transition of the CdSe host peaked at $\sim 461 \mathrm{~nm}$ is resonant with a narrow band-edge emission (BE, shaded in blue) at $\sim 465 \mathrm{~nm}$ (fwhm of $\sim 14 \mathrm{~nm}$ ). The absorption and emission spectra of undoped $3 \mathrm{ML}$ CdSe CQWs presented in Figure S1 demonstrate that the exciton energy in the CQW host is not affected by the copper dopants. ${ }^{27,29,30}$ Considering that the u-CQWs absorb significantly at the $\mathrm{BE}$ range of $\mathrm{d}$-CQWs (Figure $1 \mathrm{~b}$ ), this suggests 

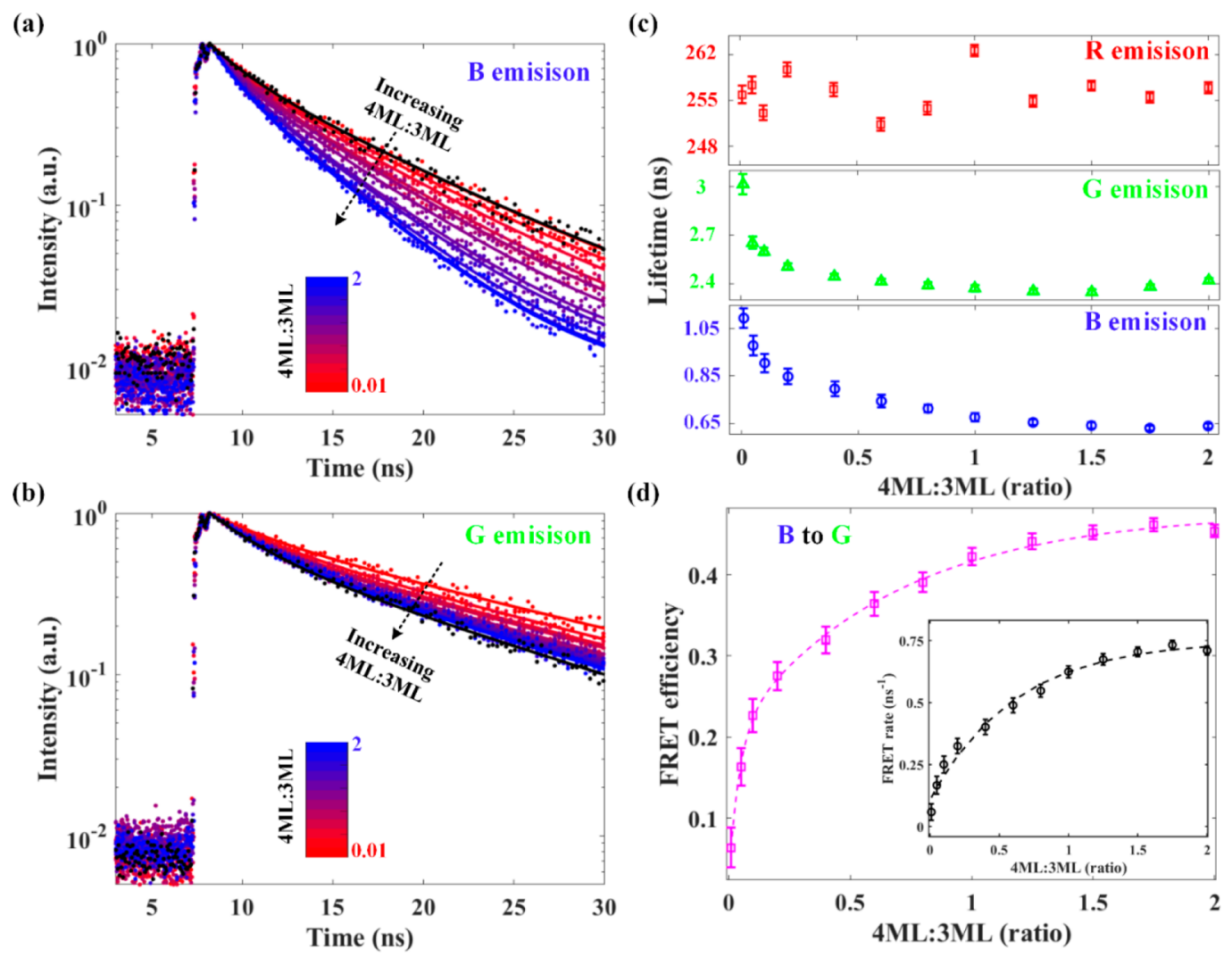

Figure 2. Dynamic characterizations of the CQW complex for FRET. Laser pulse: $100 \mathrm{ps}, 100 \mathrm{~nJ} / \mathrm{cm}^{2}$, and $355 \mathrm{~nm}$. (a) Time-resolved PL decay of the CQW complex collected in the blue emission range (from 450 to $480 \mathrm{~nm}$ ). Dots and solid lines are the results of experiments and numerical fittings, respectively. PL decay of pure $3 \mathrm{ML}$ d-CQWs is also presented as a reference (black dots and line). (b) Time-resolved PL decay of the CQW complex collected in the green emission range (from 500 to $535 \mathrm{~nm}$ ). Dots and solid lines are the results of experiments and numerical fittings, respectively. PL decay of pure $4 \mathrm{ML} \mathrm{u}$-CQWs is also presented as a reference (black dots and line). (c) Averaged lifetime of R, G, and B emission as a function of the molar concentration ratio between $4 \mathrm{ML}$ u-CQWs and $3 \mathrm{ML}$ d-CQWs. (d) Calculated FRET (the blue-to-green) efficiency as a function of the molar concentration ratio between $4 \mathrm{ML} \mathrm{u}-\mathrm{CQWs}$ and $3 \mathrm{ML} \mathrm{d}-\mathrm{CQWs}$. Inset: the corresponding FRET rate. The dashed lines are for guiding the eyes.

that FRET between BE in d-CQWs (donor) and u-CQWs (acceptor) is possible. Furthermore, it is worth noticing that, in the absorbance spectrum of d-CQWs, there exists a broad absorption tail on the red side of the electron-heavy-hole excitonic feature. This broad absorption tail, which is nearly resonant with the broad copper-related emission (CE) in Figure 1a (shaded in red), can be assigned only to the promotion of the $\mathrm{d}$ orbital electrons in $\mathrm{Cu}^{+}$to the conduction band of CdSe host; this is expressed as $[\mathrm{Ar}] 3 \mathrm{~d}^{10}+h \nu \rightarrow$ $[\mathrm{Ar}] 3 \mathrm{~d}^{9}+\mathrm{e}$, where $h \nu$ is the energy of Cu-related absorption and e denotes an electron in the CB. ${ }^{30}$ Thus, another possible FRET between u-CQWs (donors) and CE in d-CQWs (acceptors) will occur if the CE can be directly excited by the green emission in u-CQWs. To confirm this possibility, we progressively tuned the excitation photon energy below the bandgap of host CdSe from $500 \mathrm{~nm}$ down to $590 \mathrm{~nm}$. As shown in Figure 1c, CE is still observed, indicating that $\mathrm{Cu}^{+}$is photoexcited directly to $\mathrm{Cu}^{2+}$ without the hole-trapping process. Comparing the steady-state PL properties between mixed and pure CQW films is a straightforward way to explore the possible FRET between d-CQWs and u-CQWs (optically clear and uniform films for various mixture ratios are critical; see the details of film preparation in Methods). ${ }^{22,34}$ When the excitation energy matches the electron-light-hole transition of d-CQWs ( $435 \mathrm{~nm}$, top panel of Figure 1d), emission from the mixed film reveals an increase in the intensity of the $\mathrm{BE}$ in
u-CQWs while accompanied by a decrease in the intensity of BE in d-CQWs. Similarly, when we excite at the electronlight-hole transition of u-CQWs $(\sim 480 \mathrm{~nm}$, bottom panel of Figure 1d), quenching of the BE in u-CQWs and enhancement of the CE in d-CQWs are observed in the mixed film. Together, these are the first evidence that mutual FRET (Figure 1e) occurs in this CQW complex, where the excitation energy transfers from d-CQWs' excitons to u-CQWs' excitons and then back to the copper dopants in d-CQWs.

To further confirm the proposed mutual FRET and determine the rate/efficiency, time-resolved PL is conducted. We prepared 12 mixed film samples on the quartz substrates with different molar ratios between u-CQWs and d-CQWs (denoted as $4 \mathrm{ML}: 3 \mathrm{ML}$ hereafter), in addition to the reference samples of pure $\mathrm{u}-\mathrm{CQW}$ s and pure d-CQWs.

As shown in Figure 2a, trPL of BE in d-CQWs becomes shorter-lived with greater $4 \mathrm{ML}: 3 \mathrm{ML}$, indicating that a newly built-up relaxation channel in blue emission is enhanced with increasing $\mathrm{u}-\mathrm{CQW}$ concentration in the mixed film. In the case of decay kinetics for u-CQWs (Figure 2b), the lifetime is observed to be more prolonged with smaller $4 \mathrm{ML}: 3 \mathrm{ML}$, suggesting that green exciton feeding becomes more significant with increasing d-CQW concentration in the mixed film. The faster PL dynamics in the BE of d-CQWs (donor) combined with the slower PL kinetics in u-CQWs (acceptor) is the characteristic signature of FRET, ${ }^{2,22}$ and this indicates the 
(a)

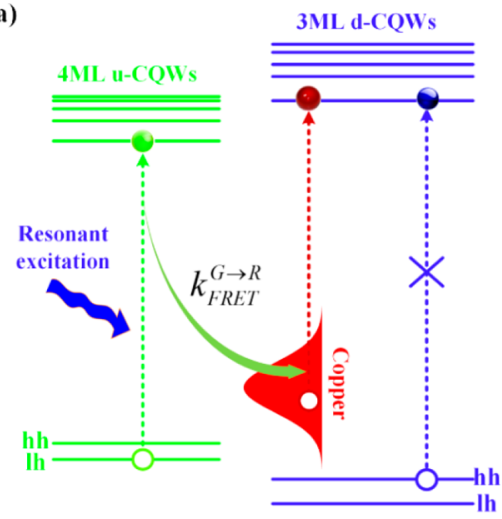

(c)

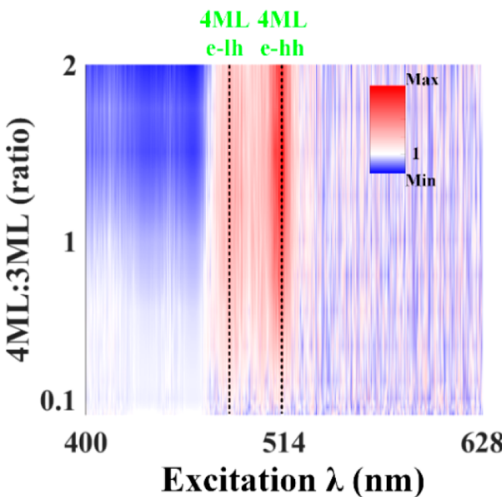

(b)

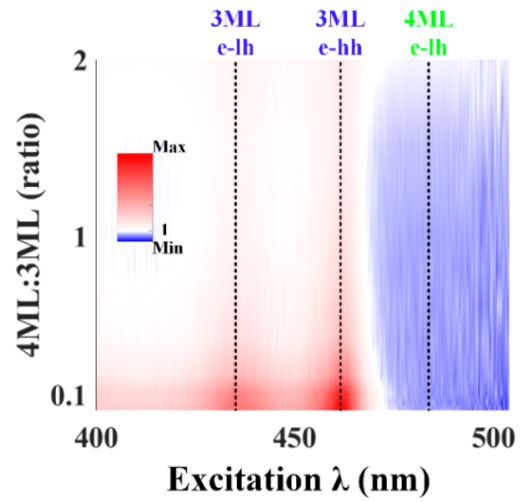

(d)

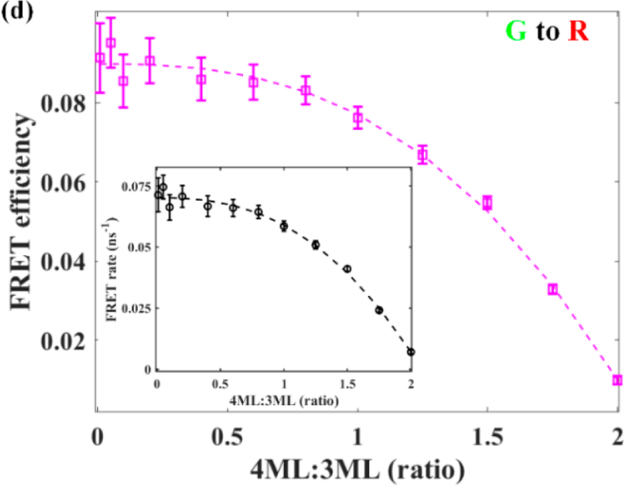

Figure 3. PLE spectroscopy of the CQW complex. (a) Schematic illustration of the working principle to characterize the FRET from excitons in 4 ML u-CQWs to dopants in $3 \mathrm{ML}$ d-CQWs; hh, heavy hole; lh, light hole. (b) 2D PLE enhancement map for the green emission (probed at $\sim 514$ $\mathrm{nm}$ ). The three dashed lines indicate the absorption transition features. Red is coded for the region with an enhancement factor greater than 1 ; blue is coded for the region with an enhancement factor less than 1 ; white is coded for the region with an enhancement factor of 1 . (c) $2 \mathrm{D}$ PLE enhancement map for the red emission (probed at $\sim 628 \mathrm{~nm}$ ). The two dashed lines indicate the absorption transition features in $4 \mathrm{ML}$ u-CQWs. The same color coding for the map as illustrated in Figure 3b. (d) Calculated FRET (from the green-to-red) efficiency as a function of the molar concentration ratio between $4 \mathrm{ML}$ u-CQWs and $3 \mathrm{ML}$ d-CQWs. Inset: the corresponding FRET rate. The dashed lines are for guiding the eyes.

outflow of energy from the blue to the green. Because of the multiple recombination channels in ensemble CQWs, the PL dynamics exhibits a multiple-exponential behavior, ${ }^{13,23,24,26}$ and an amplitude-weighted average lifetime $\left(\tau_{\mathrm{av}}\right)$ is adopted to quantify the observed differences in the decay kinetics (see all the fitting parameters for lifetime with different molar ratios in Tables S1-S3). As shown in Figure $2 \mathrm{c}, \tau_{\mathrm{av}}$ of blue emission does not change noticeably when $4 \mathrm{ML}: 3 \mathrm{ML}$ is greater than 1.5 , indicating a saturation of FRET between the donoracceptor pair. Subsequently, using the fitted $\tau_{\mathrm{B}}^{\mathrm{mix}}$ for the mixed film and $\tau_{\mathrm{B}}^{\text {pure }}$ for the pure d-CQW film, the efficiency $\left(\eta_{\mathrm{FRET}}^{\mathrm{B} \rightarrow \mathrm{G}}\right)$ and rate $\left(k_{\mathrm{FRET}}^{\mathrm{B} \rightarrow \mathrm{G}}\right)$ of the blue-to-green FRET for all molar ratios can be calculated as follows:

$$
\begin{aligned}
& k_{\mathrm{FRET}}^{\mathrm{B} \rightarrow \mathrm{G}}=k_{\mathrm{B}}^{\mathrm{mix}}-k_{\mathrm{B}}^{\text {pure }}=\frac{1}{\tau_{\mathrm{B}}^{\text {mix }}}-\frac{1}{\tau_{\mathrm{B}}^{\text {pure }}} \\
& \eta_{\mathrm{FRET}}^{\mathrm{B} \rightarrow \mathrm{G}}=1-\frac{\tau_{\mathrm{B}}^{\text {mix }}}{\tau_{\mathrm{B}}^{\text {pure }}}=1-\frac{k_{\mathrm{B}}^{\text {pure }}}{k_{\mathrm{FRET}}^{\mathrm{B} \rightarrow \mathrm{G}}+k_{\mathrm{B}}^{\text {pure }}}
\end{aligned}
$$

where $k_{\mathrm{B}}^{\mathrm{mix}}$ and $k_{\mathrm{B}}^{\text {pure }}$ are the blue emission rates in mixed and pure film, respectively. From Figure $2 d$, it can be seen that the efficiency of the blue-to-green FRET increases with increasing molar ratios of u-CQWs and then reaches a saturated level of $\sim 48 \%$. Compared to the theoretical prediction of FRET efficiency in CQWs, which is greater than 95\%, the limited efficiency in our experiments is mainly because it is not possible to have all CQWs intermixed (i.e., cofacial donor- acceptor pairs: DAD or ADA), but instead there are self-mixed CQWs (i.e., only donor or acceptor assemblies, DDD or AAA). ${ }^{22,23}$

The characterizations of the one-way FRET process (from the blue to green) in the CQW complex are obvious as presented above; however, it is hard for us to identify the FRET process from the u-CQWs to copper dopants in $d$ CQWs with the trPL measurements. There are two major reasons for this: (i) The PL decay of green emission is a convolution of the delayed acceptor kinetics (FRET from the blue to green) and accelerated donor dynamics (FRET from the green to red). In Figure 2c, we can observe that when 4 ML:3 ML is greater than 1.5 (when the blue-to-green FRET is saturated), the lifetime of green emission slows down again. This behavior implies that green emission excitons play multiple roles in the FRET process because when the amount of donors (u-CQWs) increases, the acceleration of green emission decay will be typically suppressed. ${ }^{34}$ (ii) The PL kinetics of red emission (Figure 2c) does not exhibit the delayed dynamics as expected for acceptors because the $\mathrm{CE}$ in d-CQWs originates from the radiative recombination of $\mathrm{CB}$ electrons in the CdSe host to the localized intragap states of copper and offers a long lifetime of $\sim 250 \mathrm{~ns}$. As a result, the FRET process with a fast rate may not be able to make a noticeable change to the CE dynamics (see the measured PL decay of CE with different 4 ML:3 ML ratios in Figure S2). 
To characterize the second FRET process (from green to red), photoluminescence excitation (PLE) spectroscopy across exciton absorption resonances is conducted. The different PLE intensity of the CQW complex compared to that of pure CQW film is widely used to extract the FRET information. ${ }^{35-37}$ Here, assuming the change of PLE intensity is solely attributed to the FRET process, a model of single-exciton dynamics (due to low excitation intensity in PLE measurement) is used to estimate the FRET efficiency $\left(\eta_{\mathrm{FRET}}\right)$ and rate $\left(k_{\mathrm{FRET}}\right)$ from the enhancement factors of PLE spectra (defined as the PLE intensity ratio between the mixed film and the pure film with the same amount of CQWs of interest), shown as follows:

$$
\begin{aligned}
& k_{\mathrm{FRET}}=\left(\frac{I^{\text {pure }}}{I^{\text {mix }}}-1\right) k^{\text {pure }} \\
& \eta_{\mathrm{FRET}}=1-\frac{k^{\text {pure }}}{k^{\text {pure }}+k_{\mathrm{FRET}}}=1-\frac{I^{\text {mix }}}{I^{\text {pure }}}
\end{aligned}
$$

where $I^{\text {pure }}$ and $I^{\mathrm{mix}}$ are the PL intensity of the donor excited at a wavelength of choice (typically, exciton features are selected), respectively; $k^{\text {pure }}$ is the recombination rate of pure donors. Details of the derivation of FRET rate and efficiency are described in Supplementary Note 1. This model is also used to calculate the blue-to-green FRET (see Figure S3) and compared to the results presented in Figure 2d; the good agreement of these two results using PLE and trPL spectroscopy validates our model.

More importantly, the complex can be excited at a preferred wavelength to enable only the green-to-red FRET process. As shown in Figure 3a, the CQW complex is resonantly excited at the energy of the light-hole-electron exciton of $\mathrm{u}$-CQWs ( $\sim 80 \mathrm{~nm}$, which is below the bandgap of d-CQWs), and as a result, the blue-to-green FRET will not be triggered and pure green-to-red FRET can be characterized. Figure $3 \mathrm{~b}$ shows the color plot of PLE enhancement with different molar ratios probed at the energy of green excitons $(\sim 514 \mathrm{~nm})$. The individual PLE enhancement spectra are shown in Figure S4. In contrast to the PLE of pure u-CQWs, enhanced emission intensity (red, enhancement factor: $>1$ ) is observed at the wavelength corresponding to the electron-heavy-hole $(\sim 461$ $\mathrm{nm})$ and electron-light-hole $(\sim 435 \mathrm{~nm})$ transitions in $\mathrm{d}$ CQWs, signifying the blue-to-green FRET. The more attractive observation for us is the quenched PL intensity (blue, enhancement factor: <1) located at the wavelength around the electron-heavy-hole transition of u-CQWs $(\sim 480 \mathrm{~nm})$. Furthermore, as shown in Figure 3c, the PLE enhancement map probed at the red emission wavelength $(\sim 638 \mathrm{~nm}$, the individual PLE enhancement spectra is shown in Figure S5) exhibits two clear enhancement regions exactly located at the electron-heavy-hole $(\sim 480 \mathrm{~nm})$ and electron-light-hole $(\sim 512 \mathrm{~nm})$ transitions in $\mathrm{u}$-CQWs. These two observations unambiguously indicate that the exciton energy in u-CQWs is outflowing into the copper-dopants in d-CQWs (the green-tored FRET). Therefore, in Figure 3b, we can characterize the FRET through the emission intensity change excited at the electron-heavy-hole transition of u-CQWs $(\sim 480 \mathrm{~nm})$ using the established model. The calculated efficiency and rate of green-to-red FRET are presented in Figure 3d. The efficiency of the green-to-red FRET is below $10 \%$ because of the small absorption cross section of direct dopant transition (excite $\mathrm{d}$ electrons of $\mathrm{Cu}^{+}$to the conduction band of CdSe host), as reported by Gamelin and co-workers. ${ }^{29,38}$
In summary, we have demonstrated the first example of how doped CQWs can be used to achieve a mutual FRET in a binary CQW complex. In the complex composed of $3 \mathrm{ML} d-$ CQWs and $4 \mathrm{ML}$-CQWs, distinct two-way energy-transfer routes in which the d-CQWs and u-CQWs serve both as donors and acceptors simultaneously have been presented in detail through analyzing the steady-state emission intensity, time-resolved PL, and PLE spectroscopies. These findings provide a promising approach beyond the unidirectional limitation for applications in various fields, such as biophysics, by offering a cross-check configuration to precisely probe the dynamics in biomolecules, and energy optoelectronics, through enabling more external control parameters. Future efforts in this direction lie in the improvement of mutual FRET efficiency that can enhance the signal-to-noise ratio for FRET-based fluorescence probing and expand the tunability for an external stimulus.

\section{METHODS}

Material Synthesis. Three ML Cu-doped CdSe CQWs are synthesized according to our previously published method. ${ }^{27}$ Undoped $4 \mathrm{ML}$ CdSe CQWs are also synthesized according to previously published literature. $^{23,33}$ All synthesized samples are cleaned with ethanol to remove excess ligands and are finally dispersed in hexane for further experiments and measurements.

Steady-State Optical Characterization of $u$-CQWs and $d$ CQWs. Absorption spectra of CQWs in hexane are measured by an ultraviolet-visibles spectrophotometer (Shimadzu, UV1800). PL of CQWs in hexane and PLE spectra of CQWs in solid films are recorded using a spectrofluorophotometer (Shimadzu, RF-5301PC, excitation wavelength: $355 \mathrm{~nm}$ ). Quantum yield (QY) of CQWs in hexane is measured with an integrating sphere and calculated as the ratio of absolute photon numbers of emission to absorption. The accuracy of the QY measurement is verified using Rhodamine 6G, whose QY of $94.3 \%$ measured in our setup is found to be in good agreement with the standard value of $95 \%$.

Preparation of the Solid CQW Films. The $3 \mathrm{ML}$ d-CQWs and $4 \mathrm{ML}$-CQWs dissolved in hexane are prepared to have the same molar concentration $\left(10^{-4} \mathrm{~mol} / \mathrm{L}\right)$. Solutions having different 4 ML:3 ML molar ratios are mixed using ultrasonication for $5 \mathrm{~min}$. The $1.5 \times 1.5 \mathrm{~cm}^{2}$ quartz substrates are cleaned using detergent, deionized water, acetone, and ethanol. After the substrates are dried in an oven at $80{ }^{\circ} \mathrm{C}$ for $30 \mathrm{~min}$, spin coating (at $1500 \mathrm{rpm}$ for $2 \mathrm{~min}$ ) is adopted to ensure optically clear (minimum scattering) and uniform solid films. The same solution concentration and spin-coating recipe are important to have the same film thickness and morphology for different 4 ML:3 ML molar ratios.

PL Dynamics Measurement. The time-resolved PL spectroscopy is performed in a confocal system with a single-photon avalanche photodiode (from Micro Photon Devices) and a time-correlated single-photon-counting acquisition module (Edinburgh Instruments, TCC900). For the excitation, we used a pulsed diode laser at $355 \mathrm{~nm}$ with a repetition rate of 20 $\mathrm{MHz}$ while we filtered the emission according to the three emission bands from the CQW complex. For all time-resolved PL measurements, the temporal resolution is 200 ps. All measurements are performed at room temperature in air. 


\section{ASSOCIATED CONTENT}

\section{S Supporting Information}

The Supporting Information is available free of charge on the ACS Publications website at DOI: 10.1021/acs.jpclett.9b01939.

Properties of $\mathrm{Cu}$-doped and undoped $3 \mathrm{ML} \mathrm{CdSe}$ CQWs (Figure S1), trPL of CE (red emission) in dCQWs (Figure S2), blue-to-green FRET characterization from PLE (Figure S3), separated PLE enhancement spectra (Figures S4 and S5), fitting parameters of trPL curves (Tables S1-S3), and estimation of FRET rate from PLE spectroscopy (PDF)

\section{AUTHOR INFORMATION}

\section{Corresponding Authors}

*E-mail: volkan@stanfordalumni.org.

*E-mail: hcdang@ntu.edu.sg.

\section{ORCID}

Manoj Sharma: 0000-0001-5215-9740

Muhammad Danang Birowosuto: 0000-0002-9997-6841

Hilmi Volkan Demir: 0000-0003-1793-112X

Cuong Dang: 0000-0001-6183-4082

\section{Author Contributions}

C.D. and H.V.D. led and supervised all aspects of the research. J.Y. and C.D. initiated the idea. J.Y., H.V.D, and C.D. wrote the manuscript; M.S. performed the d-CQW and u-CQW synthesis and optimized them to achieve the best performance. S.D. helped in material synthesis and characterizations. J.Y. and M.D.B performed the PL dynamics measurement; J.Y. conducted the PLE measurement. J.Y. and C.D. built up the model to characterize FRET from PLE spectroscopy. All authors analyzed the data, discussed the results, commented on the manuscript, and participated in manuscript revision.

\section{Notes}

The authors declare no competing financial interest.

\section{ACKNOWLEDGMENTS}

We acknowledge financial support from Singapore Ministry of Education through AcRF Tierl grant (MOE2017-T1-002-142) and Singapore National Research Foundation under the Program of NRF-NRFI-2016-08. H.V.D. gratefully acknowledges additional financial support from the TUBA.

\section{REFERENCES}

(1) Jares-Erijman, E. A.; Jovin, T. M. FRET imaging. Nat. Biotechnol. 2003, 21, 1387-1395.

(2) Guzelturk, B.; Demir, H. V. Near-Field Energy Transfer Using Nanoemitters For Optoelectronics. Adv. Funct. Mater. 2016, 26, $8158-8177$.

(3) Marx, V. Probes: FRET sensor design and optimization. Nat. Methods 2017, 14, 949-953.

(4) Shcherbakova, D. M.; Cox Cammer, N. C.; Huisman, T. M.; Verkhusha, V. V.; Hodgson, L. Direct multiplex imaging and optogenetics of Rho GTPases enabled by near-infrared FRET. Nat. Chem. Biol. 2018, 14, 591-600.

(5) Ben-Johny, M. B.; Yue, D. N.; Yue, D. T. Detecting stoichiometry of macromolecular complexes in live cells using FRET. Nat. Commun. 2016, 7, 13709.

(6) Li, J.; Cushing, S. K.; Meng, F.; Senty, T. R.; Bristow, A. D.; Wu, $\mathrm{N}$. Plasmon-induced resonance energy transfer for solar energy conversion. Nat. Photonics 2015, 9, 601-607.
(7) Gupta, V.; Bharti, V.; Kumar, M.; Chand, S.; Heeger, A. J. Polymer-Polymer Förster Resonance Energy Transfer Significantly Boosts the Power Conversion Efficiency of Bulk-Heterojunction Solar Cells. Adv. Mater. 2015, 27, 4398-4404.

(8) Serin, J. M.; Brousmiche, D. W.; Fréchet, J. M. J. A FRET-Based Ultraviolet to Near-Infrared Frequency Converter. J. Am. Chem. Soc. 2002, 124, 11848-11849.

(9) Chen, Y.; Zhang, L.; Graf, L.; Yu, B.; Liu, Y.; Kochs, G.; Zhao, Y.; Gao, S. Conformational dynamics of dynamin-like MxA revealed by single-molecule FRET. Nat. Commun. 2017, 8, 15744.

(10) Singh, D.; Wang, Y.; Mallon, J.; Yang, O.; Fei, J.; Poddar, A.; Ceylan, D.; Bailey, S.; Ha, T. Mechanisms of improved specificity of engineered Cas9s revealed by single-molecule FRET analysis. Nat. Struct. Mol. Biol. 2018, 25, 347-354.

(11) Raja, A.; Castillo, A. M.; Zultak, J.; Zhang, X.-X.; Ye, Z.; Roquelet, C.; Chenet, D. A.; Zande, A. M. V. D.; Huang, P.; Jockusch, S.; Hone, J.; Reichman, D. R.; Brus, L. E.; Heinz, T. F. Energy Transfer from Quantum Dots to Graphene and $\mathrm{MoS}_{2}$ : The Role of Absorption and Screening in Two-Dimensional Materials. Nano Lett. 2016, 16, 2328-2333.

(12) Prasai, D.; Klots, A. R.; Newaz, A.; Niezgoda, J. S.; Orfield, N. J.; Escobar, C. A.; Wynn, A.; Efimov, A.; Jennings, G. K.; Rosenthal, S. J.; Bolotin, K. I. Electrical Control of near-Field Energy Transfer between Quantum Dots and Two-Dimensional Semiconductors. Nano Lett. 2015, 15, 4374-4380.

(13) Olutas, M.; Guzelturk, B.; Kelestemur, Y.; Gungor, K.; Demir, $\mathrm{H}$. V. Noncontact temperature probing: highly efficient nonradiative energy transfer from colloidal semiconductor quantum dots to wells for sensitive noncontact temperature probing. Adv. Funct. Mater. 2016, 26, 2891-2899.

(14) Andrew, P.; Barnes, W. Förster Energy Transfer in an Optical Microcavity. Science 2000, 290 (2000), 785-788.

(15) Blum, C.; Zijlstra, N.; Lagendijk, A.; Wubs, M.; Mosk, A. P.; Subramaniam, V.; Vos, W. L. Nanophotonic Control of the Förster Resonance Energy Transfer Efficiency. Phys. Rev. Lett. 2012, 109, 203601.

(16) Zhang, Q.-W.; Li, D.; Li, X.; White, P. B.; Mecinović, J.; Ma, X.; Ågren, H.; Nolte, R. J. M.; Tian, H. Multicolor Photoluminescence Including White-Light Emission by a Single Host-Guest Complex. J. Am. Chem. Soc. 2016, 138, 13541-13550.

(17) Deng, R.; Qin, F.; Chen, R.; Huang, W.; Hong, M.; Liu, X. Temporal full-colour tuning through non-steady-state upconversion. Nat. Nanotechnol. 2015, 10, 237-242.

(18) Ithurria, S.; Tessier, M. D.; Mahler, B.; Lobo, R. P. S. M.; Dubertret, B.; Efros, A. Colloidal Nanoplatelets with Two-Dimensional Electronic Structure. Nat. Mater. 2011, 10, 936-941.

(19) Mahler, B.; Nadal, B.; Bouet, C.; Patriarche, G.; Dubertret, B. Core/Shell Colloidal Semiconductor Nanoplatelets. J. Am. Chem. Soc. 2012, 134, 18591-18598.

(20) Bose, S.; Shendre, S.; Song, Z.; Sharma, V. K.; Zhang, D. H.; Dang, C.; Fan, W.; Demir, H. V. Temperature-dependent optoelectronic properties of quasi-2D colloidal cadmium selenide nanoplatelets. Nanoscale 2017, 9 (19), 6595-6605.

(21) Gao, Y.; Li, M.; Delikanli, S.; Zheng, H.; Liu, B.; Dang, C.; Sum, T. C.; Demir, H. V. Low-Threshold Lasing from Colloidal $\mathrm{CdSe} / \mathrm{CdSeTe}$ Core/Alloyed-Crown Type-II Heteronanoplatelets. Nanoscale 2018, 10, 9466-9475.

(22) Rowland, C. E.; Fedin, I.; Zhang, H.; Gray, S. K.; Govorov, A. O.; Talapin, D. V.; Schaller, R. D. Picosecond Energy Transfer and Multiexciton Transfer Outpaces Auger Recombination in Binary CdSe Nanoplatelet Solids. Nat. Mater. 2015, 14, 484-489.

(23) Guzelturk, B.; Olutas, M.; Delikanli, S.; Kelestemur, Y.; Erdem, O.; Demir, H. V. Nonradiative Energy Transfer in Colloidal CdSe Nanoplatelet Films. Nanoscale 2015, 7, 2545-2551.

(24) Yeltik, A.; Olutas, M.; Sharma, M.; Gungor, K.; Demir, H. V. Nonradiative Energy Transfer between Doped and Undoped Flat Semiconductor Nanocrystals of Colloidal Quasi-2D Nanoplatelets. J. Phys. Chem. C 2019, 123, 1470-1476. 
(25) Erdem, O.; Gungor, K.; Guzelturk, B.; Tanriover, I.; Sak, M.; Olutas, M.; Dede, D.; Kelestemur, Y.; Demir, H. V. OrientationControlled Nonradiative Energy Transfer to Colloidal Nanoplatelets: Engineering Dipole Orientation Factor. Nano Lett. 2019, 19, 42974305.

(26) Guzelturk, B.; Erdem, O.; Olutas, M.; Kelestemur, Y.; Demir, H. V. Stacking in Colloidal Nanoplatelets: Tuning Excitonic Properties. ACS Nano 2014, 8, 12524-12533.

(27) Sharma, M.; Gungor, K.; Yeltik, A.; Olutas, M.; Guzelturk, B.; Kelestemur, Y.; Erdem, T.; Delikanli, S.; McBride, J. R.; Demir, H. V. Near-Unity Emitting Copper-Doped Colloidal Semiconductor Quantum Wells for Luminescent Solar Concentrators. Adv. Mater. 2017, 29, 1700821.

(28) Sharma, M.; Olutas, M.; Yeltik, A.; Kelestemur, Y.; Sharma, A.; Delikanli, S.; Guzelturk, B.; Gungor, K.; McBride, J. R.; Demir, H. V. Understanding the Journey of Dopant Copper Ions in Atomically Flat Colloidal Nanocrystals of CdSe Nanoplatelets Using Partial Cation Exchange Reactions. Chem. Mater. 2018, 30, 3265-3275.

(29) Hughes, K. E.; Hartstein, K. H.; Gamelin, D. R. Photodoping and Transient Spectroscopies of Copper-Doped CdSe/CdS Nanocrystals. ACS Nano 2018, 12, 718-728.

(30) Pinchetti, V.; Di, Q.; Lorenzon, M.; Camellini, A.; Fasoli, M.; Zavelani-Rossi, M.; Meinardi, F.; Zhang, J.; Crooker, S. A.; Brovelli, S. Excitonic Pathway to Photoinduced Magnetism in Colloidal Nanocrystals with Nonmagnetic Dopants. Nat. Nanotechnol. 2018, 13, $145-151$.

(31) Pradhan, N.; Das Adhikari, S.; Nag, A.; Sarma, D. D. Luminescence, plasmonic, and magnetic properties of doped semiconductor nanocrystals. Angew. Chem., Int. Ed. 2017, 56, $7038-7054$.

(32) Yu, J.; Sharma, M.; Li, M.; Hernandez-Martinez, P. L.; Delikanli, S.; Sarma, A.; Altintas, Y.; Hettiarachchi, C.; Sum, T.; Demir, H. V.; Dang, C. Sustained biexciton emission in colloidal quantum wells assisted by dopant-host interaction. 2019, arXiv: 1905.11571.

(33) Olutas, M.; Guzelturk, B.; Kelestemur, Y.; Yeltik, A.; Delikanli, S.; Demir, H. V. Lateral Size-Dependent Spontaneous and Stimulated Emission Properties in Colloidal CdSe Nanoplatelets. ACS Nano 2015, 9, 5041-5050.

(34) Kagan, C. R.; Murray, C. B.; Nirmal, M.; Bawendi, M. G. Electronic Energy Transfer in CdSe Quantum Dot Solids. Phys. Rev. Lett. 1996, 76, 1517-1520.

(35) Kozawa, D.; Carvalho, A.; Verzhbitskiy, I.; Giustiniano, F.; Miyauchi, Y.; Mouri, S.; Castro Neto, A. H.; Matsuda, K.; Eda, G. Evidence for fast interlayer energy transfer in $\mathrm{MoSe}_{2} / \mathrm{WS}_{2}$ heterostructures. Nano Lett. 2016, 16, 4087-4093.

(36) Chow, C. M.; Yu, H.; Jones, A. M.; Yan, J.; Mandrus, D. G.; Taniguchi, T.; Watanabe, K.; Yao, W.; Xu, X. Unusual ExcitonPhonon Interactions at van der Waals Engineered Interfaces. Nano Lett. 2017, 17, 1194-1199.

(37) Brumberg, A.; Diroll, B. T.; Nedelcu, G.; Sykes, M. E.; Liu, Y.; Harvey, S. M.; Wasielewski, M. R.; Kovalenko, M. V.; Schaller, R. D. Material Dimensionality Effects on Electron Transfer Rates Between $\mathrm{CsPbBr}_{3}$ and CdSe Nanoparticles. Nano Lett. 2018, 18, 4771-4776.

(38) Nelson, H. D.; Hinterding, S. O. M.; Fainblat, R.; Creutz, S. E.; Li, X.; Gamelin, D. R. Mid-Gap States and Normal vs Inverted Bonding in Luminescent $\mathrm{Cu}+-$ and $\mathrm{Ag}+-$ Doped CdSe Nanocrystals. J. Am. Chem. Soc. 2017, 139, 6411-6421. 